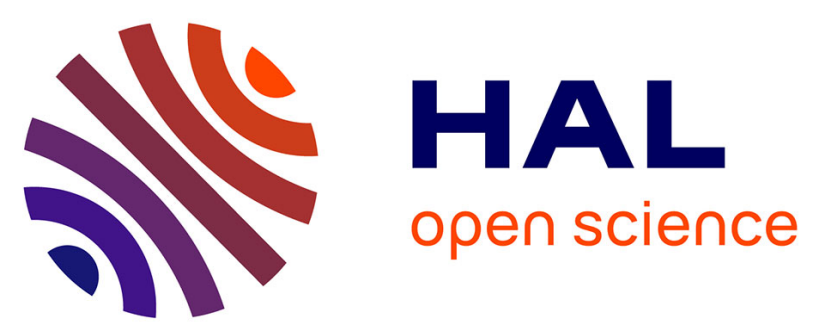

\title{
CHARACTERISTICS OF MARINE ATMOSPHERIC BOUNDARY LAYER ROLL VORTICES FROM SENTINEL-1 SAR WAVE MODE
}

Chen Wang, Alexis Mouche, Ralph Foster, Douglas Vandemark, Justin Stopa, Pierre Tandeo, Nicolas Longepe, Bertrand Chapron

\section{To cite this version:}

Chen Wang, Alexis Mouche, Ralph Foster, Douglas Vandemark, Justin Stopa, et al.. CHARACTERISTICS OF MARINE ATMOSPHERIC BOUNDARY LAYER ROLL VORTICES FROM SENTINEL-1 SAR WAVE MODE. IGARSS 2019: International Geoscience and Remote Sensing Symposium, Jul 2019, Yokohama, Japan. 10.1109/IGARSS.2019.8900287 . hal-02276981

\section{HAL Id: hal-02276981 https://imt-atlantique.hal.science/hal-02276981}

Submitted on 3 Sep 2019

HAL is a multi-disciplinary open access archive for the deposit and dissemination of scientific research documents, whether they are published or not. The documents may come from teaching and research institutions in France or abroad, or from public or private research centers.
L'archive ouverte pluridisciplinaire HAL, est destinée au dépôt et à la diffusion de documents scientifiques de niveau recherche, publiés ou non, émanant des établissements d'enseignement et de recherche français ou étrangers, des laboratoires publics ou privés. 


\title{
CHARACTERISTICS OF MARINE ATMOSPHERIC BOUNDARY LAYER ROLL VORTICES FROM SENTINEL-1 SAR WAVE MODE
}

\author{
Chen Wang ${ }^{1,2}$, Alexis Mouche ${ }^{1}$, Ralph C. Foster ${ }^{3}$, Douglas Vandemark ${ }^{4}$, Justin E. Stopa ${ }^{5}$, \\ Pierre Tandeo ${ }^{2}$, Nicolas Longepe ${ }^{6}$, Bertrand Chapron ${ }^{1}$ \\ ${ }^{1}$ IFREMER, Univ. Brest, CNRS, IRD, LOPS, Brest, France \\ ${ }^{2}$ IMT Atlantique, Lab-STICC, UBL, Brest, France \\ ${ }^{3}$ Applied Physics Laboratory, University of Washington, Seattle, USA \\ ${ }^{4}$ Ocean Processes Analysis Laboratory, University of New Hampshire, New Hampshire, USA \\ ${ }^{5}$ Department of Ocean Resources and Engineering, University of Hawaii at Manoa, Hawaii, USA \\ ${ }^{6}$ Space and Ground Segment, Collecte Localisation Satellites (CLS), Plouzane, France
}

\begin{abstract}
Millions of wave mode synthetic aperture radar (SAR) images are routinely acquired by the ESA's two sentinel-1 satellites every month over the open ocean. These SAR images capture clear imprints of atmospheric boundary layer (ABL) roll vortices. This provides a new and unique opportunity to investigate the characteristics of ABL rolls globally and statistically. In this study, we take advantage of the deep learning classification tool that has been successfully developed to automatically identify ABL rolls. For each SAR image classified with ABL rolls, roll wavelength and orientation are extracted through spectral analysis. Surface meteorological variables are also collocated with each SAR image to address the atmospheric conditions of roll occurrence. Results show that roll vortices are prevalent over the whole ocean and mainly occur in unstable to near-neutral stratification. Roll characteristics follow the theoretical expectation and are in good agreement with previous studies.
\end{abstract}

Index Terms - Marine atmospheric boundary layer, roll vortices, synthetic aperture radar, Sentinel-1 wave mode

\section{INTRODUCTION}

Roll vortices are a common feature of the turbulent atmospheric boundary layer (ABL) in near-neutral to moderately unstable stratification. They can be generated either by thermal instability, when the layer is heated from below or cooled from above, or by dynamic instability, when the wind velocity changes with the height $[1,2]$. This organized secondary circulation usually spans the whole depth of the ABL and is

We gratefully acknowledge ESA and ECMWF for Sentinel-1 and ERA5 data access and the supporting of this study by Sentinel-1A Mission Performance Center (4000107360/12/I-LG), S1-4SCI Ocean Study (4000115170/15/I-SBo), CNES (TOSCA program, COWS project) and NASA Physical Oceanography grant (NNX17AH17G). C. Wang acknowledges PhD. financial support from the China Scholarship Council. approximately aligned with the mean wind direction. It contributes significantly to the vertical transport of momentum, heat and moisture. When roll vortices present, overturning circulation commonly exists with alternating linear regions of enhanced upward and downward perturbation flows forming between the counter-rotating roll circulations. These perturbations in the ABL change the near surface winds significantly and thus modulate the sea surface roughness. Therefore, imprints of roll vortices can be captured by high resolution synthetic aperture radar (SAR) images, visible as twodimensional quasi-periodic patterns $[3,4,5,6]$.

Extensive observational, experimental, theoretical, and numerical investigations have indicated that typical roll wavelengths and orientations are 2 to $5 \mathrm{~km}$ and within $+/-20 \mathrm{deg}$ of the surface wind direction and ABL stratification is a strong indicator of their presence [1, 2, 7]. Although SAR has been proven to be a useful tool to study ABL rolls over the world's ocean $[8,9]$, at present, there is no systematic analysis dedicated to their statistical properties $[4,6,9]$. This study exploits the global Sentinel-1 (S-1) WaVe mode (WV) SAR data for investigation of ABL roll features. The geophysical classification tool created by a deep learning approach is applied to automatically identify WV SAR images of roll vortices $[10,11]$. Section 2 of this paper documents the S-1 WV classification tool and collocated meteorological variables. In section 3, we extract roll wavelength and orientation through spectral analysis and discuss the statistical results of roll characteristics as well as atmospheric conditions of roll occurrence. A summary follows in section 4 .

\section{ROLL VORTICES ON S-1 WV IMAGES}

S-1 is a constellation of two C-band SAR satellites (A \& B) that were launched by European Space Agency (ESA) in 2014 and 2016, respectively. The WV was originally designed for directional wave spectra retrieval as part of the operational 

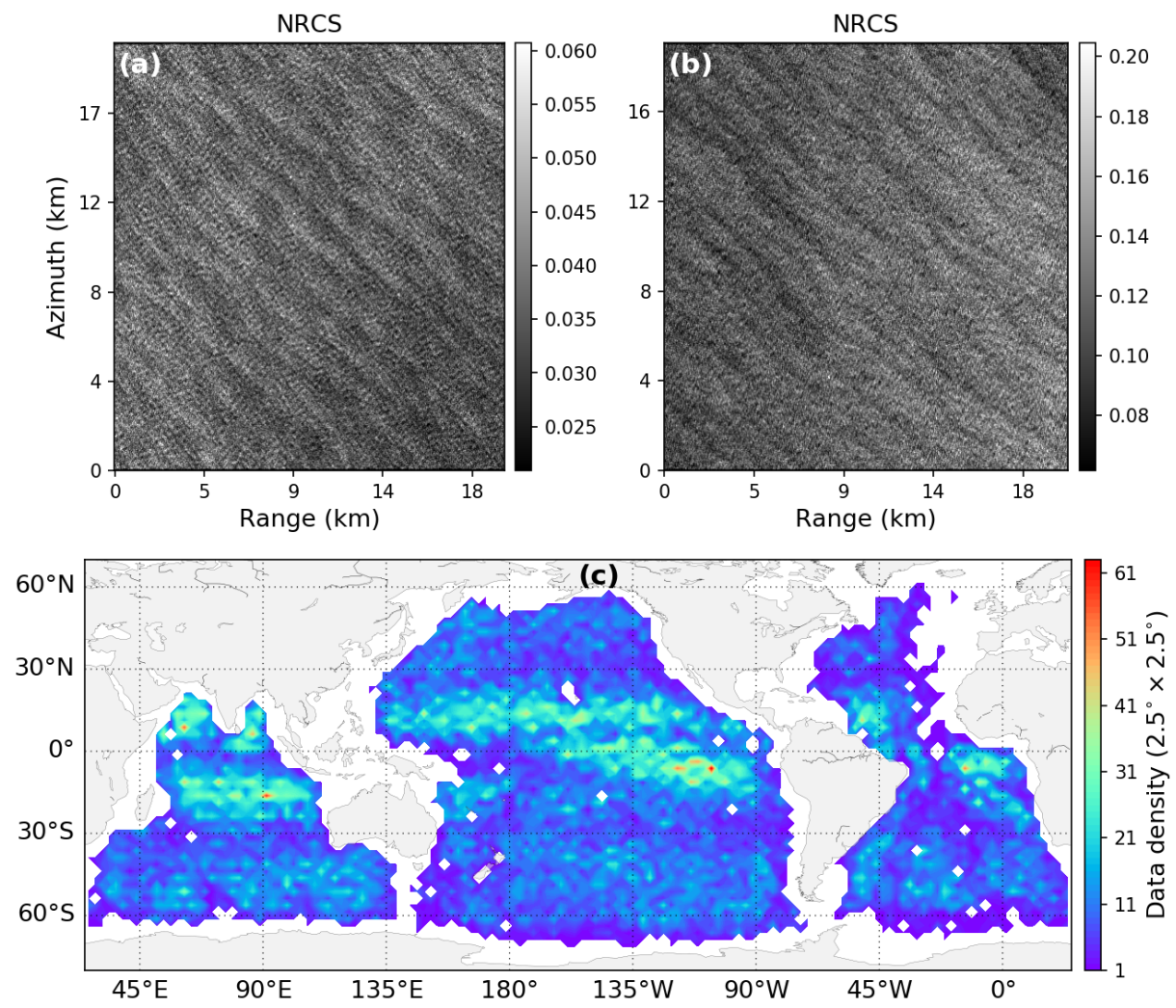

Fig. 1. Sea surface roughness examples (a,b) of ABL rolls taken by S-1 SAR WV. Panel (c) exhibits the global distribution of identified ABL rolls, color donates image count in $2.5^{\circ}$ by $2.5^{\circ}$ grid boxes.

level-2 ocean product. It acquires SAR images routinely over the open ocean at alternating incidence angles of $23^{\circ}$ (WV1) and $36.5^{\circ}$ (WV2) in VV polarization (default). Each WV SAR image has a footprint of $20 \mathrm{~km}$ by $20 \mathrm{~km}$ with a spatial resolution of $5 \mathrm{~m}$. Recent exploration of these WV SAR data reveals that $\mathrm{ABL}$ roll signatures are also well imaged [12]. Two such images are shown in Figure 1 (a) and (b). It is clear that the periodic patterns of roll vortices are superimposed on ocean wave signatures. These features are distinct enough to be identified visually or automatically using pattern recognition image processing techniques.

A geophysical classification model for the WV SAR data, called CMwv, has been successfully developed by fine-tuning the Inception-v3 deep convolutional neural network [11]. This tool is able to distinguish ten pre-defined geophysical phenomena. Specifically, the identification of ABL rolls using CMwv has better performance for WV2 compared to WV1 images with precision and recall of $96 \%$ and $83 \%$, respectively. Therefore, we focus on the WV2 SAR images in this study and use S-1A data obtained in 2016. Figure 1 (c) displays the global distribution of identified ABL rolls, counted in each $2.5^{\circ}$ by $2.5^{\circ}$ grid box. In general, roll vortices are present across the ocean as expected. In particular, they are more prevalent in the tropics compared to the ex- tratropics with a higher concentration of ABL rolls located alongside rainfall belts. We expect that the deeper convection common to the tropics is responsible for the higher occurrence of the ABL roll vortices in the tropics. Moreover, the accompaniment of rolls with rainfall is commonly observed in the hurricane boundary layer [7]. Although there is no WV acquisition in the Arctic Ocean, closed seas and coastal areas, the huge available WV SAR data set $(\sim 120,000$ per month) provides a new and unique opportunity to statistically investigate ABL physics at scales of $0.8-6 \mathrm{~km}$, that almost only SAR can achieve.

To address the atmospheric conditions of roll occurrence, we collocated each SAR image in time and space with surface meteorological variables from the European Centre for Medium-Range Weather Forecasts (ECMWF) Re-Analysis (v5) ERA5, listed in Table 1. The wind speed at $10 \mathrm{~m}$ elevation under neutral stability $\left(U_{10}\right)$, air-sea temperature difference $\left(\Delta_{T}\right)$ and bulk Richardson number $\left(R i_{B}\right)$ are examined in this work because ABL dynamics are governed by wind shear and thermal convection. These three parameters are all surface based (10 m height) and calculated using the COARE 3.0 algorithm. 
Table 1. Meteorological variables from ECMWF ERA5

\begin{tabular}{ccc}
\hline Short name & Long name & Unit \\
\hline u10 & 10 metre U wind component & $\mathrm{m} / \mathrm{s}$ \\
v10 & 10 metre V wind component & $\mathrm{m} / \mathrm{s}$ \\
sst & sea surface temperature & $\mathrm{K}$ \\
t2m & 2 metre temperature & $\mathrm{K}$ \\
d2m & 2 metre dew-point temperature & $\mathrm{K}$ \\
sp & surface pressure & $\mathrm{Pa}$ \\
blh & boundary layer height & $\mathrm{m}$ \\
\hline
\end{tabular}

\section{CHARACTERISTICS OF ROLL VORTICES}

Considering that the imprints of roll vortices on SAR images are periodic signatures, spectral analysis is effectively extracts the horizontal wavelength and orientation $[4,6]$. This technique is applicable to $\mathrm{S}-1 \mathrm{WV}$ images because roll signatures normally dominate the whole $20 \mathrm{~km}$ scenes. The estimation algorithm we used is modified from the spectral estimation unit (see details in [13]). The periodogram process is removed to include the larger wavelength signatures. In Figure 2, the two spectra estimated from the SAR images displayed in Figure 1 (a) and (b) are shown. For convenience, they have been converted from Cartesian to Polar coordinates with $\phi$ denoting roll orientation relative to the azimuth direction. Although we have interpolated the spectrum during the Cartesian to Polar conversion, the spectral resolution does not improve as shown in the Figure 2 (c) and (d). To avoid signatures of very low frequency waves and ocean waves, we concentrate on wavelengths between 0.8 and $6 \mathrm{~km}$, the black vertical lines in Figure 2.

The most energetic spectral peaks can be detected by searching for local maxima within the spectral densities from 0.8 to $6 \mathrm{~km}$. Given the peak location, marked as black stars in Figure 2, we can easily extract the roll wavelength $(\lambda)$ and orientation $\left(\phi_{\text {rolls }}\right)$. Note that $\phi_{\text {rolls }}$ needs to be converted form the image coordinate to the geographic coordinate. Then, the aspect ratio $(A R=\lambda / h, h$ is the ABL height) and angle deviation of roll orientation from the surface wind direction $\left(\Delta \phi=\left(\phi_{u 10}-\phi_{\text {rolls }}\right) * \operatorname{sign}(\right.$ lat $\left.)\right)$ can be calculated. The sign of latitude is considered in the calculation of $\Delta \phi$ to combine the southern and northern hemisphere together. The positive (negative) of $\Delta_{\phi}$ is indicative of cyclonic (anticyclonic) rotation.

Figure 3 displays the probability density functions (PDFs) of (a) wavelength, (c) aspect ratio and (d) angle deviation. Although the imprecise SAR spectral resolution limits our ability to obtain a smooth PDF of roll wavelength, $\lambda$ distribution is clearly in the lognormal. The best fitted curve for $A R$ is also the lognormal and curve for $\Delta_{\phi}$ is normal. These findings are consistent with statistical results from hurricane boundary layer [14]. The median and mean values of aspect ratio is close to 2.83 , agreeing with the theoretical expectation

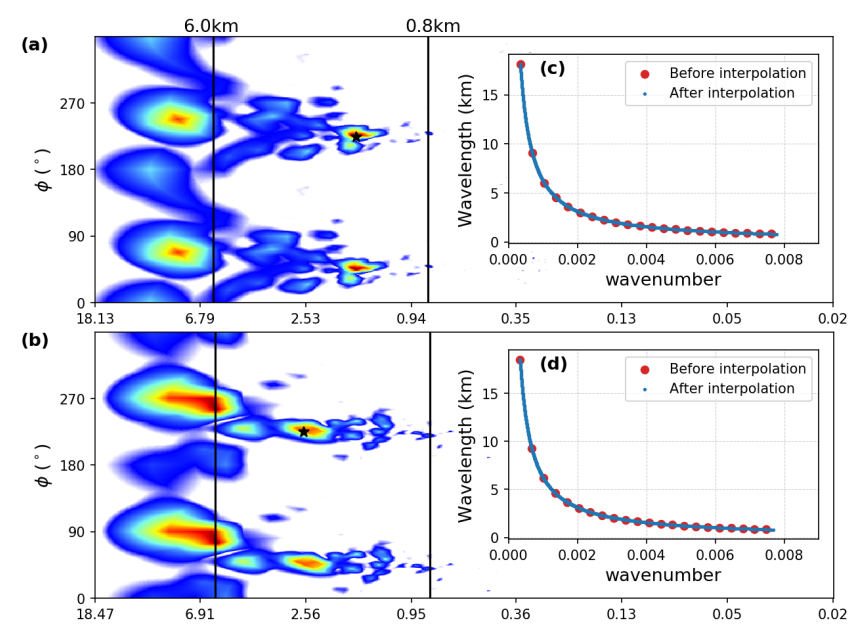

Fig. 2. Spectral analysis to extract the wavelength and orientation of ABL rolls from SAR images. (a) and (b) correspond to the images displayed in Figure 1. (c) and (d) demonstrate the spectral resolution before and after interpolation. The black vertical lines indicate the wavelength of 0.8 and 6 $\mathrm{km}$. Black stars are indicative of the detected spectral peaks.

[2], indicating good agreement between SAR observation and ABL theory. However, the positive angle deviation on Figure 3 (c) means roll orientation is on the left (right) of the surface wind direction in the northern (southern) hemisphere is unexpected [2]. This is an ongoing research that could be related to many factors such as ABL height, Coriolis force, and temperature gradients.

PDFs of wind speed, air-sea temperature difference and the bulk Richardson number are also given in Figure 3. It is reasonable that roll vortices mostly occur in medium winds conditions $(5-15 \mathrm{~m} / \mathrm{s})$, as wind shear is one of principal forming forces. As expected, ABL rolls generally exist in unstable to near-neural atmospheric conditions, corresponding to the negative $\Delta_{T}$ and $R i_{B}$. It is worth noting that there is a small portion of rolls observed under stable atmospheric conditions, which will be investigated in the future study.

\section{SUMMARY}

In this work, we have conducted preliminary investigation of ABL roll using the global S-1 WV SAR data. Global distribution of identified roll vortices proves that rolls exist everywhere and dominate the non-rainfall areas in the tropics. The bulk Richardson number indicates that rolls mostly occur in, as expected, the moderately unstable to near-neutral atmospheric conditions. Rolls aspect ratio has values of $3.39 \pm$ 1.79 (mean \pm standard deviation), which are in a lognormal distribution with geometric standard deviation of 0.5. This is in good agreement with the ABL theory predication and previous statistical results. However, the roll orientation has been found unexpected on the opposite side of surface wind 

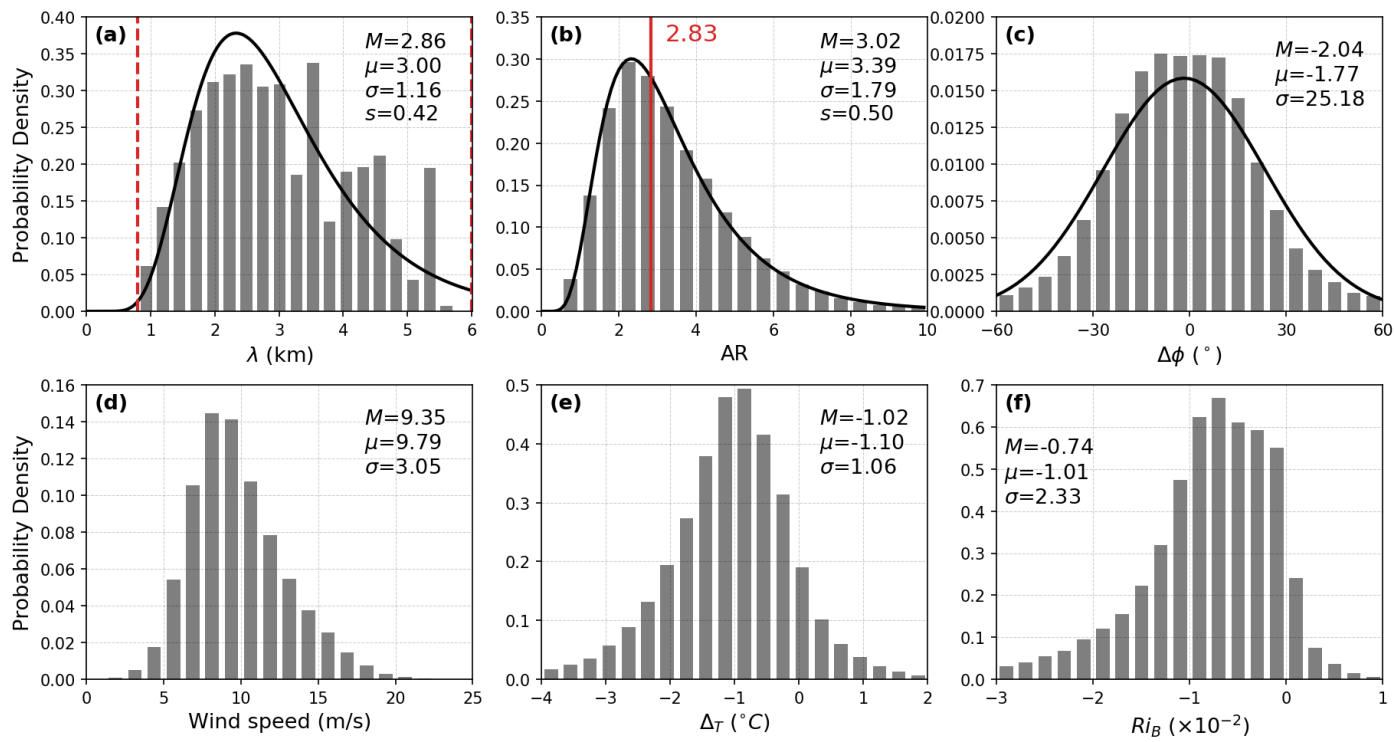

Fig. 3. Probability density functions of (a) wavelength $(\lambda)$, (b) aspect ratio $(A R)$, (c) angle deviation of orientation relative to surface wind direction $(\Delta \phi)$, (d) surface wind speed $\left(U_{10}\right)$, (e) air-sea temperature difference $\left(\Delta_{T}\right)$ and (f) the bulk Richardson number $\left(R i_{B}\right) . M, \mu, \sigma$ and $s$ are the median, mean, standard deviation and geometric standard deviation, respectively. Curve fits suggest that data in (a) and (b) are lognormal distribution, and in (c) are normal distribution.

direction. Further analysis of the positive angle deviation and other relevant studies are in progress.

\section{REFERENCES}

[1] D. Etling and R. A. Brown, "Roll vortices in the planetary boundary layer: A review," Boundary-Layer Meteorology, vol. 65, no. 3, pp. 215-248, 1993.

[2] George S. Young, David A R Kristovich, Mark R. Hjelmfelt, and Ralph C. Foster, "Rolls, streets, waves, and more: A review of quasi-two-dimensional structures in the atmospheric boundary layer," Bulletin of the American Meteorological Society, vol. 83, no. 7, pp. 997-1001, 2002.

[3] T. W. Gerling, "Structure of the surface wind field from the seasat sar," Journal of Geophysical Research: Oceans, vol. 91, no. C2, pp. 2308-2320, 1986.

[4] W. Alpers and B. Brümmer, "Atmospheric boundary layer rolls observed by the synthetic aperture radar aboard the ERS-1 satellite," Journal of Geophysical Research: Oceans, vol. 99, no. C6, pp. 12613-12621, 1994.

[5] D. Vandemark, P. D. Mourad, S. A. Bailey, T. L. Crawford, C. A. Vogel, J. Sun, and B. Chapron, "Measured changes in ocean surface roughness due to atmospheric boundary layer rolls," Journal of Geophysical Research: Oceans, vol. 106, no. C3, pp. 4639-4654, mar 2001.

[6] Xiaofeng Li, Weizhong Zheng, Xiaofeng Yang, Jun A Zhang, William G Pichel, and Ziwei Li, "Coexistence of Atmospheric Gravity Waves and Boundary Layer Rolls Observed by SAR*," Journal of the Atmospheric Sciences, vol. 70, no. 11, pp. 34483459, 2013.

[7] Ralph C. Foster, "Why Rolls are Prevalent in the Hurricane Boundary Layer," Journal of the Atmospheric Sciences, 2005.
[8] Robert A. Brown, "Serendipity in the use of satellite scatterometer, SAR, and other sensor data," Johns Hopkins APL Technical Digest (Applied Physics Laboratory), vol. 21, no. 1, pp. 21-26, 2000.

[9] Gad Levy, "Boundary layer roll statistics from SAR," Geophysical Research Letters, vol. 28, no. 10, pp. 1993-1995, may 2001.

[10] C. Wang, A. Mouche, P. Tandeo, J. Stopa, B. Chapron, R. Foster, and D. Vandemark, "Automated geophysical classification of sentinel-1 wave mode sar images through deep-learning," in IGARSS 2018 - 2018 IEEE International Geoscience and Remote Sensing Symposium, July 2018, pp. 1776-1779.

[11] C. Wang, A. Mouche, P. Tandeo, J. Stopa, V. Gressani, D. Vandemark, R. Foster, and B. Chapron, "Automated classification of Sentinel-1 SAR vignettes provides nearly global data for ocean surface process studies," Remote Sensing of Environment, 2019 (in preparation).

[12] C. Wang, A. Mouche, P. Tandeo, J. Stopa, N. Longp, G. Erhard, R. Foster, D. Vandemark, and B. Chapron, "A labeled Ocean SAR Imagery Dataset of Ten Geophysical Phenomena from Sentinel-1 Wave Mode," Geoscience Data Journal, 2018 (submitted).

[13] Harald Johnsen and Fabrice Collard, "Sentinel-1 ocean swell wave spectra (osw) algorithm definition," Tech. Rep. S1-TNNRT-52-7450, Norut, Troms $\varnothing, 2011$.

[14] Ian Morrison, Steven Businger, Frank Marks, Peter Dodge, and Joost A. Businger, "An Observational Case for the Prevalence of Roll Vortices in the Hurricane Boundary Layer," Journal of the Atmospheric Sciences, vol. 62, no. 8, pp. 2662-2673, 2005. 